\section{A atenção primária à saúde nos países da União Européia: configurações e reformas organizacionais na década de 1990}

\author{
Primary care in European Union countries: \\ configurations and organizational reforms \\ in the 1990s
}

Ligia Giovanella ${ }^{1}$

Impulsionadas por contextos econômicos restritivos e propósitos de contenção de gastos em saúde, reformas organizacionais dos serviços de atenção primária foram implantadas em diversos países da União Européia durante a década de 1990, com o objetivo de promover a coordenação dos serviços prestados entre os diversos níveis de atenção, resultando em novos arranjos organizacionais. Afora propósitos de controle de gastos, uma melhor coordenação dos cuidados busca responder a exigências de mudanças no perfil epidemiológico com crescente prevalência de doenças crônicas. $\mathrm{O}$ cuidado de doentes crônicos resulta em interdependência interorganizacional, pois estes pacientes utilizam simultaneamente serviços de diversas complexidades, exigindo a coordenação entre níveis de atenção.

Os objetivos do presente trabalho são examinar as configurações institucionais do primeiro nível de atenção à saúde nos países da União Européia (composta por 15 países até maio de 2004) e analisar as reformas organizacionais da atenção ambulatorial que objetivaram melhor coordenação dos cuidados, em parte destes países, durante a década de 1990. A partir de revisão bibliográfica, análise documental e entrevistas com informantes-chave, apresenta-se perfil da estrutura da atenção primária nos países da União Européia; são examinadas as principais medidas propostas e discutidos condicionantes da implantação das reformas organizacionais.
Primary Health Care; Health Sector Reform Health Services; Health Services Administration 
No Brasil, a necessidade de melhor organização do sistema de atenção com a constituição de redes integradas e sistemas funcionais de atenção à saúde tem sido motivo de intenso debate político e acadêmico 1,2 . Ponto central neste debate são mudanças no modelo assistencial, especialmente aquelas pretendidas na atenção básica/atenção primária impulsionadas por incentivos federais para a implementação do Programa Saúde da Família (PSF) 3,4,5. A revisão aqui apresentada sobre as configurações do primeiro nível de atenção e de recentes reformas pró-coordenação em países centrais com sistemas públicos de atenção à saúde consolidados, tem por propósito contribuir para este debate brasileiro indicando um leque de alternativas disponíveis e desafios ainda presentes na atenção primária naqueles países. A análise de experiências de outros países, consideradas as peculiaridades do desenvolvimento histórico-cultural dos sistemas de proteção social em cada país, pode contribuir para debates nacionais ainda que as experiências não possam ser reproduzidas, dados seus condicionantes históricos, institucionais, políticos e sociais 6

O artigo está organizado em quatro partes. A primeira seção inicia com a explicitação do sentido em que o termo "atenção primária em saúde” é empregado neste artigo. Em seguida, apresenta-se panorama da atenção primária em saúde nos países da União Européia com destaque para características estruturais. Na segunda seção, identificam-se as principais medidas pró-coordenação implementadas nos países estudados, com destaque para Reino Unido e Alemanha, agrupando-as em dois conjuntos de mecanismos organizacionais inter-relacionados: aquelas que proporcionaram maior poder e controle da atenção primária sobre prestadores de outros níveis, e, aquelas que expandiram o leque de serviços ofertados no primeiro nível. Analisa-se a seguir alguns condicionantes das diferentes trajetórias das reformas observadas nos países, relacionadas ao legado institucional e à estrutura sócio-política setorial. Nas considerações finais, discutem-se perspectivas de reorganização do sistema de saúde com fortalecimento da atenção primária, indicando a tensão entre o exercício de práticas gerenciais ampliadas de coordenação pelo "general practitioner" (generalista) e sua credibilidade frente aos pacientes e outros prestadores.

\section{Panorama do primeiro nível de atenção à saúde na Europa Ocidental}

A atenção ambulatorial de primeiro nível, isto é, os serviços de primeiro contato do paciente com o sistema de saúde, direcionados a cobrir as afecções e condições mais comuns, e resolver a maioria dos problemas de saúde de uma população são em geral denominados de serviços de atenção primária. Não há, contudo, uniformidade no emprego do termo atenção primária à saúde ("primary health care"), identificando-se pelo menos três linhas de interpretação. Pode referir-se a: (i) programa seletivo com cesta restrita de serviços; (ii) um dos níveis de atenção correspondendo aos serviços ambulatoriais médicos de primeiro contato não-especializados 7 , incluindo ou não amplo espectro de ações de saúde pública e de serviços clínicos direcionados a toda a população; (iii) ou de forma abrangente, a uma concepção de modelo assistencial e de organização do sistema de saúde como trabalhado por Starfield 8 .

Nos países europeus, o termo atenção primária refere-se, de modo geral, aos serviços ambulatoriais de primeiro contato, diferente do que se observa nos países periféricos, nos quais atenção primária com freqüência corresponde também a programas seletivos, focalizados e de baixa resolutividade para cobrir determinadas necessidades previamente definidas de grupos populacionais em extrema pobreza 1,9.

A definição de Starfield 8 para atenção primária é assumida por autores na Europa 10,11 e contempla: a prestação de serviços de primeiro contato, a assunção de responsabilidade longitudinal pelo paciente (continuidade da relação clínico-paciente, ao longo da vida) independente da ausência ou presença de doença, a garantia de cuidado integral a partir da consideração dos âmbitos físicos, psíquicos e sociais da saúde dentro dos limites de atuação do pessoal de saúde, e, coordenação das diversas ações e serviços necessários para resolver necessidades menos freqüentes e mais complexas. Nesta concepção de "primary health care community oriented", os serviços de atenção primária requerem estar orientados para a comunidade conhecendo suas necessidades de saúde, centrar-se na família para bem avaliar como responder às necessidades e ter competência cultural para reconhecer as diferentes necessidades dos grupos populacionais 8 .

Nos países europeus, a ênfase do primeiro nível de assistência está na clínica e nos cuidados individuais, sejam estes preventivos ou curativos, e, em geral há acordo entre os "policy makers" europeus de que a atenção primária 
deve ser a base de um sistema de saúde bem desenhado e orientar a organização do sistema como um todo 10,12,13,14. No presente artigo, o termo atenção primária é empregado em sentido lato, referindo-se aos serviços ambulatoriais de primeiro contato, independente da concepção do modelo assistencial e do modo de organização do sistema de atenção à saúde do país, uma vez que a atenção ambulatorial de primeiro nível é prestada nos países da União Européia com uma grande variação de configurações institucionais, seja em sua estrutura em termos de organização e financiamento, seja nas práticas realizadas. Nos países da União Européia observam-se diferenciados mecanismos de financiamento e pagamento, gama de profissionais envolvidos, espectro de serviços clínicos oferecidos, posição e papel do profissional médico de atenção primária no sistema 15,16,17,18.

Entre as características estruturais do primeiro nível de atenção do sistema de saúde dos países da União Européia serão aqui sucintamente descritas: os principais mecanismos de financiamento, a posição dos serviços de atenção primária no sistema de saúde identificando-se os profissionais responsáveis pelo primeiro contato, e o exercício da função de porta de entrada/"gatekeeper", a vinculação institucional dos profissionais de atenção primária e as modalidades de sistema de pagamento aos generalistas.

\section{Mecanismos de financiamento}

As principais modalidades de financiamento dos sistemas de saúde, incluindo o financiamento da atenção primária em saúde, são condicionadas pelos modelos de proteção social. Os sistemas de saúde conformados historicamente nesses países europeus podem ser agrupados em dois modelos de proteção principais: bismarckiano, em base a seguros sociais de saúde ("Social Health Insurance" - SHI) de contratação compulsória financiados por contribuições vinculadas ao trabalho assalariado (sete países) e, o modelo beveridgiano de serviços nacionais de saúde ("National Health Service" - NHS) ordenados por autoridades estatais e financiados com recursos fiscais (oito países) 19. Nos SHI, o asseguramento ao risco de adoecer não é de responsabilidade direta da administração estatal. Esta responsabilidade é transferida para instituições de direito público de administração autônoma não diretamente estatais - as caixas de seguro social de doença-, geridas por representações dos trabalhadores e empregadores. A atuação destas seguradoras públicas é regulada e controlada pelo Estado e o financiamento é realizado de modo solidário por empregadores e trabalhadores mediante contribuições compulsórias proporcionais aos salários 20.

Nos serviços nacionais de saúde, o acesso é universal, independente da posição na ocupação, entendendo-se a assistência à saúde como direito de cidadania e garantindo-se acesso sem necessidade de contribuição prévia direta com financiamento por meio de recursos fiscais. Neste modelo, a presença estatal é importante com oferta predominantemente pública nos setores hospitalar e ambulatorial, contudo, observa-se diferentes " $m i x$ " público privado e posições diferenciadas do profissional de atenção primária no sistema. As modalidades de financiamento da atenção primária em saúde são indissociáveis das modalidades de sistemas de saúde. Assim, entre os quinze países da União Européia (composição até maio de 2004), em oito países funciona um Serviço Nacional de Saúde, sendo a atenção primária portanto financiada com recursos fiscais provenientes de impostos gerais. Nos outros sete países operam Seguros Sociais de Saúde com atenção ambulatorial de primeiro nível, financiada por contribuições de empregadores e trabalhadores proporcionais aos salários (Tabela 1). Estas modalidades de financiamento solidário são todavia complementadas em oito dos 15 países por participação financeira direta no ato da utilização desembolsada pelos pacientes na forma de taxa de co-pagamento, da qual, em geral, grupos vulneráveis estão isentos (baixa renda, crianças, gestantes, pacientes crônicos) (Tabela 2).

\section{Posição dos serviços de atenção primária no sistema de serviços de saúde}

Um aspecto crucial para a caracterização da atenção primária é a análise da posição desses serviços na rede assistencial enquanto serviços de primeiro contato com instituição de porta de entrada obrigatória ("gatekeeper"), considerada instrumento fundamental para permitir a coordenação dos cuidados pelo clínico geral.

$\mathrm{O}$ ator principal da atenção primária é o generalista 15, ou "family doctor" ou "Hausarzt", aqui traduzido como clínico geral ou generalista. O generalista é o principal profissional médico de primeiro contato em 11 dos países da União Européia (Tabela 1), contudo, a organização dos sistemas e a posição desses profissionais e dos serviços ambulatoriais de primeiro nível na rede diferem entre países, observando-se variação quanto ao tipo de serviço e profissional responsável pelo primeiro contato dos pacientes com o sistema de saúde. 
Características dos serviços de primeiro contato nos países da União Européia.

\begin{tabular}{|c|c|c|c|c|c|}
\hline Países & $\begin{array}{l}\text { Profissional de primeiro } \\
\text { contato }\end{array}$ & $\begin{array}{l}\text { Unidade de atenção } \\
\text { primária em saúde }\end{array}$ & $\begin{array}{l}\text { Papel de } \\
\text { "gatekeeper" }\end{array}$ & $\begin{array}{l}\text { Inscrição de } \\
\text { pacientes }\end{array}$ & $\begin{array}{l}\text { Número de } \\
\text { pacientes na lista } \\
\text { dos generalistas }\end{array}$ \\
\hline \multicolumn{6}{|c|}{$\begin{array}{l}\text { Serviço Nacional } \\
\text { de Saúde }\end{array}$} \\
\hline Dinamarca & Generalista & Consultório de grupo & $x$ & $x$ & 1.600 \\
\hline Espanha & Generalista ou pediatra & Centro de saúde & $x$ & $x$ & 2.500 \\
\hline Finlândia & Generalista & Centro de saúde & Não (25\%) & Não (1/3) & - \\
\hline Grécia & Generalista & Centro de saúde & Não & Não & - \\
\hline Itália & Generalista ou pediatra & Consultório individual & $x$ & $x$ & $1.030(1.500)$ \\
\hline Portugal & Generalista & Centro de saúde & $x$ & $x$ & 1.500 \\
\hline Reino Unido & Generalista & Consultório de grupo & $x$ & $x$ & 1.850 \\
\hline Suécia & Generalista & Centro de saúde & Não & Não (opcional) & Não (2.000) \\
\hline \multicolumn{6}{|l|}{ Seguro Saúde } \\
\hline Alemanha & Generalista ou especialista & Consultório individual & Não (hosp) & Não & - \\
\hline Áustria & Generalista ou especialista & Consultório individual & Não (hosp) & Não & - \\
\hline Bélgica & Generalista & Consultório individual & Não & Não & - \\
\hline França & Generalista ou especialista & Consultório individual & Não & Não & - \\
\hline Holanda & Generalista & Consultório em grupo & $x$ & $x$ & 2.350 \\
\hline Irlanda & Generalista & Consultório individual & $x$ & $X^{\star}$ & - \\
\hline Luxemburgo & Generalista ou especialista & Consultório individual & Não & Não & - \\
\hline
\end{tabular}

Fonte: Boerma 16; European Union of General Practitioners 12, Starfield 8, European Observatory on Health Care Systems 28 , Macinko et al. 26

* Inscrição somente para pacientes de baixa renda;

** Médias de pacientes inscritos por generalista para Dinamarca, Itália, Portugal e Reino Unido; para Espanha e Holanda número máximo.

A organização da atenção ambulatorial em níveis de atenção, com serviços de primeiro contato ofertados por clínico geral para todos os cidadãos frente à maioria dos problemas de saúde, responsável pelos encaminhamentos necessários a um segundo nível especializado, é mais freqüente nos países com sistemas nacionais de saúde. Mecanismos de coordenação hierárquica, do tipo "gatekeeper", por meio do qual se delega ao generalista poder sobre outros níveis de atenção foram estabelecidos em países com serviços nacionais de saúde já nos anos 60 21, estabelecendo-se uma clara separação de papéis entre médicos generalistas e especialistas. Atualmente, em sete (cinco com NHS) dos 15 países da União Européia (Dinamarca, Itália, Portugal, Espanha, Reino Unido, Irlanda e Holanda) o generalista exerce a função de "gatekeeper", a unidade de saúde na qual trabalha é a porta de entrada obrigatória do sistema de saúde, e serve de filtro para o acesso aos serviços prestados por especialistas, constituindo-se num primeiro nível hierárquico 12,16 (Tabela 1). Nos sistemas de porta de entrada obrigatória, os generalistas têm posição protegida e controlam as referências para atenção secundária, isto é, um formulário específico expedido pelo generalista é requerido para obter acesso aos cuidados especializados. Em geral, até as reformas dos anos 1990, regras administrativas dominavam os procedimentos para referência com baixa possibilidade de escolha entre prestadores especialistas, pelos pacientes.

$\mathrm{Na}$ maior parte dos países com esquemas de seguro social (cinco em sete países) não há separação da atenção ambulatorial em níveis entre a atenção prestada por clínicos gerais e aquela prestada por especialistas -, sendo livre aos segurados a escolha entre a procura direta ao clínico geral ou ao especialista. É o que se observa na Alemanha, Áustria, França e Luxemburgo, que apresentam sistemas sem hierarquização ambulatorial, conforme ilustrado na Tabela $116,17,20,22$.

Nos países com "gatekeeper", os generalistas além de controlar amplamente as referências para especialistas também funcionam mais freqüentemente como serviço de primeiro contato para um maior elenco de situações ${ }^{23}$. No Reino Unido, Holanda e Dinamarca, os gene- 
Características selecionadas da prática profissional de generalistas e da atenção ambulatorial nos países da União Européia.

\begin{tabular}{|c|c|c|c|c|c|}
\hline Países & $\begin{array}{c}\text { \% de generalistas } \\
\text { entre profissionais } \\
\text { autônomos }\end{array}$ & $\begin{array}{c}\text { Sistema de } \\
\text { pagamento } \\
\text { (principal) }\end{array}$ & $\begin{array}{l}\text { Especialista } \\
\text { restrito ao hospital }\end{array}$ & $\begin{array}{c}\text { Co-pagamento } \\
\text { APS }\end{array}$ & $\begin{array}{c}\% \text { de generalistas } \\
\text { no total de } \\
\text { médicos }^{\star}\end{array}$ \\
\hline \multicolumn{6}{|c|}{$\begin{array}{l}\text { Serviço Nacional } \\
\text { de Saúde }\end{array}$} \\
\hline Dinamarca & 100 & Capitação + US* & $x$ & Não & 25 \\
\hline Espanha & 4 & Salário & Não & Não & $37 *$ \\
\hline Finlândia & 2 & Salário & $x$ & $x$ & $37 *$ \\
\hline Grécia & 30 & Salário (US) & Não & $x$ & $\ldots$ \\
\hline Itália & 98 & Capitação (80\%) & Não & Não & 26 \\
\hline Portugal & 1 & Salário & Não & $x$ & 23 \\
\hline Reino Unido & 99 & Capitação + US & $x$ & Não & $60 *$ \\
\hline Suécia & 1 & Salário & Parte nos CS & $x$ & 10 \\
\hline Alemanha & 100 & US (teto) & Não & $x$ & 22 \\
\hline Áustria & 99 & US (paciente) & Não & $x$ & 31 \\
\hline Bélgica & 97 & US & Não & $x$ & $46^{*}$ \\
\hline França & 97 & US (reembolso) & Não & $x$ & 54 \\
\hline Holanda & 93 & Capitação +US & $x$ & Não & $33^{*}$ \\
\hline Irlanda & 91 & Capitação & $\ldots$ & $\ldots$ & 42 \\
\hline Luxemburgo & 98 & US (reembolso) & Não & $x$ & 34 \\
\hline
\end{tabular}

Fonte: European Union of General Practitioners 12; Starfield 8; Evans 17; European Observatory on Health Care Systems 28; Rosenbrock \& Gerlinger 29.

* Percentual de generalistas entre o total de médicos em atividade segundo Starfield 8 para os outros países,

de acordo com Evans 17, em 1991; para Alemanha, Rosenbrock \& Gerlinger 29; para Suécia e Áustria (1997) dados de European Union of General Practitioners 12.

ralistas são o serviço de primeiro contato para a maior parte dos problemas de saúde independente do sexo ou idade dos pacientes, assistindo adultos, mulheres e crianças 12,24.

A responsabilidade pelo primeiro contato é compartilhada em alguns países por outros profissionais de atenção primária. Ginecologistas e pediatras compartilham com os clínicos gerais o papel de serviço de primeiro contato no atendimento dos correspondentes grupos populacionais. Na Itália e Espanha, generalistas e pediatras são responsáveis pelo primeiro nível de atenção, enquanto na Suécia esse papel é também compartilhado por enfermeiras e ginecologistas e parte das crianças é atendida por pediatra (Tabela 1). Na Inglaterra e Holanda, a atenção primária em saúde inclui além dos generalistas, dentistas, enfermeiras, visitadoras domiciliares, educadores de saúde, massagistas, fisioterapeutas, parteiras, entre outros.

A responsabilidade formal pelo primeiro contato não garante que a demanda de fato seja atendida na unidade de saúde em que traba- lha o generalista. A procura direta aos hospitais e serviços de emergência é freqüente em alguns países, nos quais um sistema de "gatekeeper" vigora formalmente. Na Espanha, estima-se que cerca de $50 \%$ dos contatos médicos são feitos nos serviços de emergência dos hospitais 25 . Em Portugal, Finlândia e Grécia, o generalista deveria ser o serviço de primeiro contato para a maioria dos problemas de saúde, contudo há procura freqüente de serviços de emergência hospitalares de modo a desviar-se do sistema de referência e acessar diretamente aos especialistas 26,27 .

O exercício da função de "gatekeeper" implica a obrigatoriedade de inscrição dos pacientes em um consultório de generalista ou serviço primário de saúde. Como se observa na Tabela 1, países com "gatekeeper" também dispõem de sistema de inscrição de pacientes. A inscrição nos serviços é realizada por iniciativa do cidadão/segurado que desfruta de alguma liberdade de escolha - e possibilidade posterior de troca -, entre profissionais de saúde ou serviços atuantes em determinado espaço geográfico, 
diferente da adscrição ou cadastramento compulsório da população como ocorre no PSF no Brasil. O tamanho das listas de cidadãos inscritos por generalista apresenta importante variação entre países: de 1.030 na Itália até no máximo de 2.500 na Espanha 12,18,28. Chama a atenção, a pequena extensão das listas de cidadãos inscritos por generalista, muito menor do que a norma estipulada no Brasil para o território de abrangência da equipe do PSF com adscrição de até mil famílias e média de 3.450 pessoas por equipe.

\section{Vinculação profissional}

e sistema de pagamento

Os generalistas quanto à sua forma de vinculação institucional ao sistema de saúde podem ser profissionais autônomos ou assalariados, posição esta também em parte relacionada com a modalidade de sistema de saúde. Em dez dos 15 países da União Européia, os clínicos gerais são profissionais autônomos. A autonomia desses profissionais é observada em todos os sete países nos quais o modelo de proteção social à saúde funda-se em seguros sociais. Por outro lado, em cinco dos oito países com serviços nacionais de saúde, a maioria dos médicos generalistas é assalariada e trabalha em centros de saúde de natureza pública (Tabela 2). Os profissionais autônomos trabalham em consultórios próprios, o que conforma nestes casos uma estrutura de oferta de atenção primária predominantemente privada. Os profissionais autônomos em geral trabalham isoladamente em consultórios individuais, com exceção do Reino Unido, Holanda e Dinamarca, países nos quais a maioria dos médicos de família trabalha em grupo 18.

O modo de inserção dos profissionais médicos no sistema de saúde condiciona em parte o sistema de pagamento a esses profissionais. Nos serviços nacionais de saúde, financiados por recursos fiscais, os generalistas são, com maior freqüência, empregados públicos (cinco países) recebendo remuneração na forma de salários. Quando profissionais autônomos (Dinamarca, Itália e Reino Unido), o sistema de pagamento tem por base um mecanismo de capitação, isto é, pagamentos per capita por paciente inscrito, em geral com alguma diferenciação das taxas para pessoas de idade mais avançada e complementação por pagamentos adicionais referentes à realização de determinados serviços que se pretenda incentivar (como medidas preventivas, por exemplo). Nos países com seguros sociais de saúde, em geral, os generalistas enquanto profissio- nais autônomos são remunerados na base de "fee-for-service", ou seja, pagamentos por unidades de serviços prestados. Cada consulta é fragmentada em uma série de subações com remuneração correspondente.

Nas últimas duas décadas observou-se tendência a hibridismo nos sistemas de pagamento, buscando-se equilibrar os incentivos das duas formas de remuneração: reduzir o risco da capitação produzir subassistência, incentivando-se ações prioritárias com pagamentos adicionais, e, controlar o estímulo à multiplicação de ações dos sistemas de pagamento "fee-for-service" ao promover a assunção de responsabilidades financeiras dos prestadores por meio de esquemas de capitação 20,22,29.

\section{Espectro de serviços ofertados pelo generalista}

Quanto à prática clínica, os países europeus diferem consideravelmente no espectro de serviços ofertados pelos clínicos gerais aos seus pacientes, seja na realização de procedimentos médicos, acompanhamento de doentes crônicos ou realização de atividades preventivas. Em geral, os generalistas estão muito envolvidos com ações curativas e na resposta a demandas individuais dos pacientes (preventivas ou curativas), sendo a educação em saúde tarefa menos desenvolvida. Nos países em que o generalista exerce a função de "gatekeeper", como anteriormente mencionado, esse profissional atende espectro mais ampliado de problemas incluindo aquele da mulher e da criança, e está mais envolvido com ações relacionadas a problemas psicossociais 23 . Nos outros países, também é freqüente o atendimento de crianças pelo generalista, contudo, a atenção à saúde da mulher fica a cargo de ginecologistas. A realização de visitas domiciliares é atividade rotineira de generalistas ainda que apresente variação importante do número de visitas realizado por semana, sendo mais freqüente entre generalistas profissionais autônomos 16. A realização de ações preventivas também apresenta grande variação. Tomando a aferição rotineira de pressão arterial, independente do tipo de queixa, como indicador da preocupação dos clínicos gerais no diagnóstico precoce e controle de doenças crônicas, estudo realizado por Boerma 16 mostrou ser esta prática freqüente na maioria dos países estudados, contudo com importante variação entre $37 \%$ na Holanda a mais de $90 \%$ no Reino Unido, França, Bélgica e Alemanha. Observa-se também variação de práticas entre os generalistas que clinicam em centros urbanos e de áreas rurais, estes últimos 
oferecem um espectro mais ampliado de serviços do que seus colegas citadinos 30 .

A prática dos generalistas também varia muito em relação ao número de consultas realizadas por um clínico ao dia (16 a 50), ao tempo/intervalo destinado na agenda do consultório à marcação de cada consulta de rotina (entre 10 e 20 minutos) 16 ou o tempo médio de cada contato com cada paciente (5 a 15 minutos) 18 .

As diferentes configurações institucionais e características da estrutura de oferta e organização da atenção ambulatorial de primeiro nível em países europeus apresentado neste panorama foram a base de diversas iniciativas recentes de reformas e condicionaram a implementação de medidas por vezes similares.

\section{Reformas organizacionais da atenção primária}

Durante a década de 1990 foram implementadas reformas organizacionais dos serviços de atenção primária em diversos países europeus, com o objetivo de promover a coordenação dos serviços prestados entre os diversos níveis de atenção, resultando em novos arranjos organizacionais de oferta desses serviços. Compartilhando contextos econômicos restritivos, com redução do ritmo de crescimento, despesas em saúde crescentes em decorrência da progressiva incorporação de onerosas tecnologias de forma desordenada e sem avaliação adequada 31 e, envelhecimento de suas populações com aumento da prevalência de doenças crônicas, governos de diversos países europeus empreenderam reformas em seus sistemas de saúde com propósitos de contenção de gastos desde a década de 1980. Ainda que as estruturas ampliadas de proteção social à saúde não tenham sido desmanteladas, a ênfase na contenção de gastos impressa por governos conservadores levou à introdução de diversas medidas restritivas para racionalização da demanda, e, de mecanismos de mercado, incentivando-se a competição entre prestadores e entre seguros sociais $32,33,34$. Pelo lado da oferta, durante os anos 90, em parte dos países, gradualmente foram implementadas reformas organizacionais que incidiram sobre o setor ambulatorial e incluíram entre seus objetivos promover a coordenação dos serviços prestados entre os diversos pontos da rede assistencial, com fortalecimento dos serviços de atenção primária. Ao final dos anos 90, com mudanças das coalizões governamentais em alguns países, entre os quais Reino Unido e Alemanha, medidas para reorganização da oferta foram inten- sificadas com objetivos de melhoria da qualidade e eficiência.

Ainda que motivadas por propósitos de eficiência, as propostas pró-coordenação acompanham mudanças no perfil epidemiológico e a introdução progressiva de ampla gama de métodos diagnósticos e terapêuticos, o que atualiza a importância do papel de coordenação dos cuidados. Reconhece-se a interdependência interorganizacional presente no cuidado de doentes crônicos uma vez que necessitam acessar simultaneamente serviços de diversas complexidades, exigindo a coordenação entre níveis de atenção; o que potencialmente garante prestações mais custo-efetivas e melhor desempenho do sistema de saúde.

As medidas implementadas pelas reformas organizacionais da atenção ambulatorial que objetivaram melhor coordenação dos cuidados em países da União Européia durante a década de 1990, podem ser agrupadas em dois principais conjuntos de mecanismos organizacionais inter-relacionados: (i) aquelas que proporcionaram maior poder e controle da atenção primária sobre prestadores de outros níveis (como coordenador ou comprador), e (ii) aquelas que expandiram o leque de funções e serviços ofertados no primeiro nível, ampliando o papel de prestador 10,21, incluindo novas ações curativas, serviços comunitários de saúde mental, "home care" ou cuidados paliativos. Além destas, as reformas de atenção primária incluíram outras medidas de apoio requeridas para o sucesso das reformas pró-coordenação, relacionadas a sistemas de informação e comunicação, e educação continuada 35,36,37.

Maior poder e controle da atenção primária sobre prestadores de outros níveis

Três principais tipos de mecanismos tiveram por objetivo aumentar o poder da atenção primária sobre os outros níveis de atenção: mecanismos de mercado ou compra, arranjos cooperativos atuando na interface de serviços primários e especializados, e, a definição de porta de entrada obrigatória ("gatekeeping") pelo clínico geral 21 .

As reformas organizacionais que proporcionariam maior grau de controle e poder aos serviços de atenção primária sobre os outros níveis de atenção em geral tiveram por base um sistema de "gatekeeping", no qual os serviços ou profissionais de atenção primária são a porta de entrada do sistema e controlam não apenas a utilização de serviços nos outros níveis, como também passaram a dispor de algum grau de autoridade sobre o custo e a qua- 
lidade da atenção especializada e hospitalar, comprando ou contratando serviços especializados para seus pacientes 10. Mecanismos de mercado possibilitaram a delegação de poder de compra aos serviços de atenção primária, sendo exemplos importantes destas iniciativas a constituição de "generalista-funholding" e atualmente as fundações de atenção primária ("primary care trust" - PCT) no Reino Unido 38, 39,40,41 e, a concessão aos distritos de atenção primária na Suécia (nos condados de Stockholm, Dalarma e Bohus) de poder de compra dos serviços hospitalares 42 .

Mecanismos de mercado que possibilitaram a delegação de poder de compra aos serviços de atenção primária tiveram algum sucesso em reduzir as filas de espera para consultas a especialistas, contudo, estudos realizados nos "generalista-fundholdings", organizações que assumiam o risco financeiro por serviços especializados e de atenção primária, criados com a política de mercado interno na primeira metade dos anos 90 no Reino Unido, indicaram que generalistas com freqüência não tinham conhecimento para direcionar adequadamente os recursos para cobrir as necessidades de seus pacientes 43 e a competição entre os " $g e$ neralista-fundholders" e as autoridades sanitárias, atuando em seus respectivos mercados, resultou em redução de poder das autoridades sanitárias e aumento de iniqüidades na utilização de serviços entre pacientes vinculados aos diferentes esquemas de prestação 38,40,41.

Arranjos cooperativos, articulando os prestadores de um mesmo nível de atenção - em parte firmados por iniciativas dos próprios profissionais de saúde - foram observados no Reino Unido paralelamente à constituição do mercado interno (abolido em 1997) e, a partir de 1999, numa revisão crítica da experiência anterior, foram constituídos pelo governo trabalhista grupos de atenção primária ("primary care groups" - PCG) transformados desde 2002 em PCT. A implementação dos PCTs significou um processo de desconcentração do NHS, conferindo-se maior autonomia aos órgãos locais, e, envolveu mecanismos de mercado e mecanismos cooperativos entre prestadores do nível primário. Os PCTs implantados pelo governo trabalhista baseiam-se nas políticas prévias dos "generalista-fundholding groups", todavia diferem da experiência anterior na medida em que são organizações públicas, exigem a participação compulsória de todos os clínicos gerais do NHS de uma determinada região geográfica, incorporam outros serviços sociais e comunitários, e, estão submetidos a diversos mecanismos de prestação de contas em termos clínicos e financeiros 22,38. Atualmente, na Inglaterra, 304 PCTs organizam o conjunto de serviços primários do NHS. Cada PCT é integrado por cerca de cinqüenta generalistas, outros serviços de atenção primária, serviços sociais e autoridades locais de saúde $38,39,40$. Os PCTs passaram a ser o órgão administrativo do NHS em nível local, responsável pela atenção à saúde da população de determinada área geográfica. Os PCTs têm três funções principais: melhorar a saúde por meio de serviços de saúde pública; credenciar e comprar serviços de saúde (hospitalares e especializados); e prover e desenvolver serviços de atenção primária e serviços comunitários. Assim, o PCT gerencia os prestadores locais - generalistas, dentistas, farmácias e optometristas -, compra serviços hospitalares (internações e especialistas) dos "NHS Hospital Trusts" e, serviços de planejamento familiar, "home care" e de visitadoras sanitárias dos "Community Health Trust" 22,39.

Outro importante exemplo de arranjos cooperativos foi observado na Alemanha a partir da segunda metade da década de 90. Pressionados por restrições orçamentárias cada vez mais fortes, os próprios médicos credenciados dos seguros sociais de doença alemães buscaram modos mais cooperativos de atuação na forma de círculos de qualidade, comunidades para compra e uso conjunto de equipamentos médicos, e redes de consultórios. Mais presentes em pequenas localidades, as redes agregam de 30 a 50 profissionais médicos do setor ambulatorial de diversas especialidades (clínicos gerais e especialistas), de determinada região sem constituir hierarquia interna. De acesso livre, aos segurados em geral, realizam amplo espectro das atividades ambulatoriais e oferecem aos seus pacientes uma atenção mais articulada entre os consultórios integrantes. Ainda que em geral frouxa, a articulação com o setor hospitalar é buscada por meio de grupos de trabalho conjuntos, discussão de protocolos de conduta e na área de prontoatendimento, instalando-se serviços organizados pelos médicos das redes em dependências de hospitais 22,44,45,46.

Também no Reino Unido foram observadas iniciativas de cooperação tomadas pelos próprios médicos, em especial na Escócia, onde, a partir de 1999, foram constituídas organizações integradas de atenção primária, as cooperativas locais de atenção à saúde de participação voluntária motivada pelo desejo de colaboração com outros prestadores e de provisão de serviços de melhor qualidade 47 . Na Dinamarca, a cooperação e a comunicação entre generalistas e hospital foram estimuladas por 
meio da contratação de generalistas em tempo parcial por hospitais, atuando na interface dos serviços primários e especializados. Este generalista exerce a função de elo de comunicação entre o hospital e o conjunto de generalistas da área. Trabalha como coordenador para o hospital ou como consultor em um dos departamentos do hospital, por exemplo, cirúrgico, e estabelece a comunicação com todos os generalistas da região em assuntos relacionados às prestações, referências e contra-referências 48 .

A introdução de sistema de "gatekeeping" foi experimentada parcialmente em alguns países com seguros sociais de saúde em meados dos anos 1990, todavia, sem muito sucesso 21. Na França, um plano com este objetivo não chegou a ser implementado, devido a fortes resistências das associações profissionais, e, na Alemanha, experiências piloto iniciadas em meados dos anos 90 não tiveram continuidade. Além da resistência das associações de médicos das caixas, os segurados foram pouco motivados a abdicar da livre escolha de prestadores a qual estão acostumados 22,49. Experiências recentes buscam implementar nova concepção de redes de clínicos gerais na Alemanha com função de "gatekeeper", que pretende o fortalecimento da coordenação e da condução dos cuidados pelo clínico geral com estreita cooperação com especialistas 22,50.

\section{Expansão do leque de funções e serviços ofertados no primeiro nível}

Um segundo conjunto de medidas das reformas organizacionais da atenção primária dos anos 90, expandiu o perfil das atividades realizadas por profissionais desse nível de atenção, incluindo novas ações curativas, serviços comunitários de saúde mental, "home care" ou cuidados paliativos, e/ou incentivou a produção de ações com oferta insuficiente especialmente medidas preventivas e de promoção da saúde. Em alguns casos os serviços de atenção primária substituíram outros prestadores (hospital ou especialista) na oferta de determinados procedimentos (pequenas cirurgias, métodos diagnósticos, reabilitação, procedimentos especializados) 10. Em outros casos, a atenção primária passou a colaborar sistematicamente com outros serviços, compartilhando um esquema de atenção com base na definição de diretrizes clínicas para doenças específicas como nos programas de gestão clínica ("disease management" - DMP), ampliando suas funções. No Reino Unido, a expansão das atividades realizadas pelos generalistas resultou dos arranjos cooperativos impulsionados pelas no- vas responsabilidades de contratação de serviços especializados e hospitalares para seus pacientes ("services commissioning"). Diversas iniciativas ampliaram o escopo das funções do primeiro nível: contratação de especialistas em tempo parcial para trabalho em centros de saúde junto com generalistas, integração com serviços de enfermagem e de saúde mental, cooperativas de generalistas para prestar prontoatendimento fora do horário comercial, e, realização de procedimentos especializados pelos próprios generalistas que se tornam referência interna para determinado grupo de generalistas. Parte dos generalistas passou a ser qualificada como "general practitioners com especial interesse", provendo serviços especializados em seus consultórios para os pacientes de seu PCT 13,22, e os PCTs podem criar serviços próprios para prestação de cuidados secundários. Avaliações preliminares, segundo Rico et al. 21, apontam resultados positivos. Serviços especializados prestados pela atenção primária apresentariam resultados de saúde similares, pelo menos para agravos freqüentes 51. Pacientes e médicos mostram-se satisfeitos com a extensão do escopo de atividades da atenção primária, pois reduz os custos de deslocamento dos pacientes e melhora as oportunidades dos generalistas expandirem conhecimentos e habilidades.

Programas de gestão clínica com compartilhamento por diversos prestadores de um esquema de atenção a partir da definição de diretrizes clínicas e fluxos de atenção para doenças específicas foram difundidos em países com seguros sociais de saúde. Na Holanda envolvem generalistas, hospitais e serviços de "home care" 21. França e Alemanha implementaram recentemente programas semelhantes. $\mathrm{Na}$ Alemanha foram implantados programas nacionais de gestão clínica para doenças crônicas, envolvendo o generalista como coordenador, especialistas e hospitais, com definição de conduta clínica e fluxo para encaminhamentos 52. Nos DMPs, o clínico geral ("Hausartz”) tem funções ampliadas e assume o papel de "gatekeeper" e de "manager": inscreve pacientes, coordena a assistência, informa o paciente e negocia objetivos de atenção, apoiado por sistema de informação e de "reminder" e, de mecanismos de educação continuada. Com base em critérios e fluxos definidos, e conforme a necessidade, encaminha para o especialista ou para o hospital, e recebe o retorno do paciente, responsabilizando-se pela coordenação dos cuidados. 


\section{Medidas similares - trajetórias distintas}

Embora certa similaridade e convergência de medidas, bem como compartilhamento de contextos similares (econômico, setorial, epidemiológico) possam ser observados, as reformas, condicionadas pelo legado institucional $\mathrm{e}$ a estrutura sociopolítica setorial, percorreram diversas trajetórias, ocorreram em ritmos diferentes, e envolveram instituições e organizações distintas 21,53.

As diferentes trajetórias das reformas de atenção primária estiveram em parte condicionadas pelo modelo de sistema de proteção social à saúde: se do tipo beveridgiano na forma de serviço nacional de saúde financiado por recursos fiscais com posição importante das autoridades estatais, ou, se do tipo bismarckiano, com base em seguros sociais de saúde de contribuição compulsória dependente da participação no mercado de trabalho, e nos quais vigoram esquemas de tomada de decisão neocorporativos com participação conjunta das autoridades estatais, profissionais e seguradoras. Nos serviços nacionais de saúde, como discutido na primeira seção, em geral os generalistas são profissionais autônomos com contrato de longo prazo e já exerciam a função de "gatekeeper" com lista de pacientes inscritos por consultório, isto é, detinham o monopólio do primeiro contato. Nesses países, as reformas pró-cooperação tiveram maior sucesso. Nos países com seguros sociais de saúde, os profissionais de atenção primária também são profissionais autônomos, contudo, em geral não exercem a função de "gatekeeper" e competem com os especialistas pelos pacientes no estabelecimento do primeiro contato. Nesta situação, em geral, os generalistas detêm menor apoio dos pacientes e têm menor poder no mercado frente a especialistas.

A função de "gatekeeper" confere poder ao generalista frente a especialistas, o que influencia nos percursos das reformas. Nos sistemas com "gatekeeping", as associações de profissionais de atenção primária são mais fortes e foram as principais aliadas dos gestores governamentais setoriais em reformas promotoras da coordenação. Jogaram papel importante no Reino Unido, Dinamarca e Holanda no desenho das reformas e facilitaram a implementação ${ }^{21}$. Nesses países, como descrito na primeira parte deste trabalho, os generalistas são profissionais autônomos com função de "gatekeeper" e organizam-se em associações relativamente fortes. Em semelhante situação, os profissionais de atenção primária têm maior possibilidade de expandir suas fun- ções, ampliando seu poder técnico - poder informal baseado no conhecimento. Por outro lado, a forte oposição de associações profissionais dominadas por especialistas em países com seguros sociais, dificultou as reformas que objetivavam fortalecer o papel do generalista como condutor da atenção na França 35 e na Alemanha 49. Nestes países, em geral as associações de profissionais de atenção primária eram fracas, os seguros sociais estiveram pouco interessados e a legislação foi hesitante. Ainda que se observem atualmente algumas mudanças no panorama assistencial e arranjos cooperativos tenham sido ensaiados por alguns seguros sociais, as experiências de cooperação receberam pouco apoio e o processo de implementação é ainda muito lento 54 .

Dificuldades de implementação decorrem também de contradições internas ao conjunto de medidas propostas em algumas reformas. Na Alemanha, a par do fortalecimento do papel do clínico geral na atenção, o objetivo principal das reformas tem sido incrementar a competição seja entre caixas seguradoras, seja entre prestadores de serviços de saúde. A introdução de mecanismos de mercado, em especial a competição, inibe a conformação de arranjos cooperativos.

\section{Considerações finais}

Com base na revisão realizada é possível afirmar que permanecem diversas configurações da atenção de primeiro nível nos países da União Européia, ainda que medidas similares pró-coordenação tenham sido formuladas. No processo de reformas, os serviços de atenção primária ampliaram suas diferentes funções clínicas, de coordenação da atenção, e, financeiras, estando em curso mudanças organizacionais na atenção primária com redefinição das funções do clínico geral como condutor dos cuidados ao paciente.

Duas questões permanecem fundamentais na análise das perspectivas de reorganização do sistema de saúde com fortalecimento do papel da atenção primária: a possibilidade de exercício da função de coordenação pelo generalista e, a credibilidade deste profissional frente aos pacientes e outros prestadores, condição sine qua non para o exercício da coordenação 55 .

O exercício da função de coordenação pelo generalista envolve a coordenação da provisão de serviços por outros prestadores no próprio nível primário de atenção (enfermagem domiciliar e sociais), bem como a coordenação dos serviços prestados por especialistas nos níveis 
secundário ou hospitalar. "Coordenadores de atenção primária ajudam a guiar o paciente através do complicado e potencialmente arriscado labirinto de especialistas e procedimentos médicos, coordenando os cuidados especializados e provendo conselhos como parte de uma relação de longo prazo e confiança mútua" 56 (p. 2046). A coordenação acarreta em exercer novas funções clínicas, mas também preponderantemente gerenciais, o que pode minar a confiança dos outros profissionais e pacientes no generalista. Com as reformas organizacionais dos anos 90, os generalistas passaram a desempenhar cada vez mais o papel de agentes estatais, controlando referências para especialistas, assumindo responsabilidades pelo orçamento de atenção primária e pela compra de serviços hospitalares para seus pacientes. $\mathrm{O}$ incremento do papel organizacional do generalista aumenta seu poder/autoridade frente a outros prestadores, mas pode afetar a confiança dos pacientes e a credibilidade dos especialistas, o que dificultaria o exercício da coordenação.

Não há plena concordância de que o generalista deva exercer isoladamente o papel de coordenador da atenção, esta função poderia ser compartilhada por outros profissionais que dividiriam funções gerenciais evitando-se a perda da credibilidade no generalista. Por outro lado, com a complexificação crescente da atenção médica é difícil para um único profissional agir como "gatekeeper" e coordenar todos os cuidados 57 . A progressiva interdependência entre níveis de atenção e serviços, decorrente da crescente relevância das doenças crônicas, aponta a necessidade de um trabalho em rede. Pacientes portadores de agravos crônicos, em geral são usuários freqüentes de diversos níveis de atenção simultaneamente. Nesta situação, arranjos organizacionais em rede baseados em tomada de decisão coletiva, na cooperação e confiança mútua seriam mais adequados para enfrentar o problema da coordenação 58.

A análise realizada aponta medidas que podem contribuir para a coordenação da atenção primária, entre elas: definir porta-de-entrada obrigatória constituída por equipe multiprofissional (que pode ser diferenciada conforme o agravo principal) responsável pela condução dos pacientes no emaranhado da rede assistencial; ampliar as atividades clínicas dos médicos de atenção primária com incentivos para a continuidade de tratamento de doentes crônicos seguindo diretrizes clínicas; implantar esquema de incentivos adequados para generalistas, pacientes e especialistas para a adesão aos novos modelos organizacionais; e equilibrar as funções clínicas e gerenciais exercidas pelo generalista.

O generalista permanece como importante ator da atenção primária, contudo, o efetivo exercício do papel de coordenação dos cuidados implica superar importante desafio de encontrar equilíbrio adequado entre as funções clínicas e gerenciais. A responsabilidade por elenco ampliado de funções clínicas aumenta seu poder técnico e credibilidade; a expansão de suas funções gerenciais incrementa seu poder administrativo mas pode corroer a confiança em sua capacidade técnica por parte de especialistas e pacientes, e reduzir a satisfação do generalista na sua prática profissional.

\section{Resumo}

Propósitos de contenção de gastos em saúde e exigências de mudanças no perfil epidemiológico, impulsionaram durante a década de 90 reformas organizacionais dos serviços de atenção primária em países da União Européia, com o objetivo de promover a coordenação dos serviços prestados pelos diversos níveis de atenção. Neste artigo, a partir de revisão bibliográfica, análise documental e entrevistas com informanteschave são analisadas as configurações institucionais do primeiro nível de atenção à saúde e discutidas as reformas organizacionais pró-coordenação nos países da União Européia. A atenção ambulatorial de primeiro nível é prestada nesses países com grande variedade de configurações institucionais, observando-se diferenciados mecanismos de financiamento, gama de profissionais envolvidos, espectro de serviços clínicos oferecidos e posição do profissional médico de atenção primária no sistema de saúde. Com as reformas da última década, ocorreu diversificação de modelos organizacionais da atenção ambulatorial com ampliação das funções clínicas, gerenciais de coordenação da atenção, e financeiras dos profissionais de atenção primária, indicando processo em transição com redefinição das funções do clínico geral como condutor dos cuidados ao paciente.

Atenção Primária em Saúde; Reforma do Setor Saúde; Serviços de Saúde; Administração de Serviços de Saúde 


\section{Agradecimentos}

Trabalho produzido com o apoio de bolsa de pósdoutorado concedida pela Coordenação de Aperfeiçoamento de Pessoal de Nível Superior do Ministério da Educação, Brasil.

\section{Referências}

1. Mendes EV. Os grandes dilemas do SUS. Salvador: Casa da Qualidade Editora; 2001.

2. Hartz Z, Contandriopoulos AP. Integralidade da atenção e integração dos serviços de saúde: desafios para avaliar a implantação de um sistema sem muros. Cad Saúde Pública 2004; 20 Suppl 2:S331-6.

3. Teixeira CF. A mudança do modelo de atenção à saúde no SUS: desatando nós, criando laços. Saúde Debate 2003; 27:257-77.

4. Escorel S, Giovanella L, Mendonça MHM, Magalhães R, Senna MCM. Avaliação da implementação do Programa Saúde da Família em dez grandes centros urbanos: síntese dos principais resultados. Brasília: Ministério da Saúde; 2002.

5. Merhy EE, Onocko R. Agir em saúde. São Paulo: Editora Hucitec; 1997.

6. Hsiao WC. Comparing health care system: what nations can learn from one another. J Health Polit Policy Law 1992; 17:570-93.

7. World Health Organization. Health care systems in transition. Production template and questionnaire. Copenhagen: World Health Organization Regional Office for Europe; 1996.

8. Starfield B. Atenção primária, equilíbrio entre necessidades de saúde, serviços e tecnologia. Brasília: Organização das Nações Unidas para a Educação, a Ciência e a Cultura/Ministério da Saúde; 2002.

9. Barrenechea JJ, Trujillo E, Chorny A. El processo SPT/2000 y sus implicaciones para la orientación de la planificación y administración de los sistemas de servicios de salud. Bogotá: Editorial Universidad de Antioquia; 1990.

10. Saltman R, Rico A, Boerma W, editors. Primary care in the driver's seat? Organizational reform in European primary care. http://www.euro. who. int/observatory/Studies/20020522_7 (acessado em Jan/2004).

11. WONCA Europe. The European definition of general practice/family practice. http://www.medisin. ntnu.no/wonca/ (acessado em Jan/2004).

12. European Union of General Practitioners. GP in Europe. http://www.uemo.org (acessado em Dez/ 2004).

13. Department of Health/National Health Service. Chief executive's report to the NHS. London: Department of Health/National Health Service; 2003.

14. Wörz M, Wismar M. Green politics in Germany: what is Green health care policy? Int J Health Serv 2001; 31:847-67.

15. Saltman R, Rico A, Boerma W, editors. Primary care in the driver's seat? Berkshire: Open University Press; 2005.
16. Boerma WGW. Profiles of general practice in Europe. Utrecht: Netherlands Institute for Health Services Research; 2003.

17. Evans PR. Medicine in Europe: the changing scene in general practice in Europe. BMJ 1994; 308: 645-8.

18. Jepson GMH. How do primary health care systems compare across Western Europe? Pharmaceutical Journal 2001; 267:269-73.

19. Immergut E. Health politics - interest and institutions in Western Europe. New York: Cambridge University Press; 1992.

20. Giovanella L. Solidariedade ou competição? Políticas e sistema de atenção à saúde na Alemanha. Rio de Janeiro: Editora Fiocruz; 2001.

21. Rico A, Saltman R, Boerma W. Organizational restructuring in European Health System: the role of primary care. Social Policy and Administration 2003; 37:592-608.

22. Giovanella L. Redes integradas, programas de gestão clínica e clínico geral-coordenador: reformas recentes do setor ambulatorial na Alemanha [Relatório de Pós-doutorado]. Frankfurt am Main: Institut für Medizinische Soziologie, JW Goethe Universität; 2004.

23. Boerma WGW, van der Zee J, Fleming DM. Service profiles of general practitioners in Europe. Br J Gen Pract 1997; 47:481-6.

24. van der Bussche $H$, Dukelberg S, Middel B, van Dijk J. Mythos und Realität des Hausarztes in den Niederlanden. Jahrbuch für Kritische Medizin 1995; 25:147-65.

25. European Observatory on Health Care Systems. Health care systems in transition: Spain. http:// www.euro.who.int/document/e70504.pdf (acessado em Nov/2004)

26. Macinko J, Starfield B, Shi L. The contribution of primary care systems to health outcomes within Organization for Economic Cooperation and Development (OECD) countries, 1970-1998. Health Serv Res 2003; 38:831-65.

27. World Health Organization. Health care systems in transition. Finland. Copenhagen: World Health Organization, Regional Office for Europe; 1996.

28. European Observatory on Health Care Systems. Health care systems in transition United Kingdom. Copenhagen: European Observatory on Health Care Systems/WHO Regional Office for Europe; 1999.

29. Rosembrock R, Gerlinger T. Gesundheitspolitik. Eine sytematische Einführung. Bern: Verlag Hans Huber; 2004.

30. Boerma WGW, Groenewegen PP, van der Zee J. General Practice in urban and rural Europe: the range of curative services. Soc Sci Med 1998; 47: 445-53. 
31. Abel-Smith B. The escalation of health care costs: How did we get there? In: Organization for Economic Cooperation and Development, editor. Health care reform. The will to change. Paris: Organization for Economic Cooperation and Development; 1996. p. 17-30.

32. Hacker JS. Dismantling the health care state? Political institutions, public policies and the comparative politics of health reform. Br J Polit Sci 2004; 34:693-724

33. Pierson P. Retrenchment and restructuring in an age of austerity: what (if anything) can be learned from the affluent democracies? Cad Saúde Pública 2002; 18 Suppl:7-11.

34. Almeida C. Reformas do Estado e reformas de sistemas de saúde. Ciênc Saúde Coletiva 1999; 4: 263-86.

35. Chevreul K, Mousquès J, Paris V. Measures to improve patterns of practice and their impact on general practitioners in France. In: $8^{\text {th }}$ Meeting The European Health Policy Group. Clinicians and System Performance 2004; Paris: CREDES; 2004.

36. Bahrs O, Gerlach FM, Szecsenyi J, Andres E. Ärztliche Qualitätszirkel. Leitfaden für den Arzt in Praxis und Klinik. Collogne: Deutscher ÄrzteVerlag; 2001.

37. Beyer M, Gerlach FM, Flies U, Grol R, Krol Z, Munck A, et al. The development of quality circles/peer review groups as a method of quality improvement in Europe. Results of a survey in 26 European countries. Fam Pract 2003; 20:44351.

38. Iliffe S, Munro J. New labour and Britain's National Health Service: an overview of current reforms. Int J Health Serv 2000; 30:309-34.

39. Bindman AB, Weiner JP, Majeed A. Primary care groups in the United Kingdom: quality and accountability. Health Aff 2001; 20:132-44.

40. Fisher P. The reform of the British National Health Service. J Public Health Policy 1998; 20:138-48.

41. Light DW. Managed care in a new key: Britain's strategies for the 1990s. Int J Health Serv 1998; 28:427-44.

42. Michelsen K. Wettbewerbsorientierte Reformen im schwedischen Gesundheitssystem der 90er Jahre. Jahrbuch für Kritische Medizin 2003; 38:3147.

43. Mechanic D. The Americanization of the British National Health Service. Health Aff 1995; 14:5167.

44. Szecsenyi J, Magdeburg K, Kluthe B, Weber C, Bausch J, Schindler H, editors. Ein praxisnetz erfolgreich gestalten. Erfahrungen und ergebnisse aus zwei jahren ärztliche qualitätsgemeinschaft ried. Göttingen: KV Hessen/VDAK/AEV/AQUA; 1999.

45. Baur R. Die erste Generation Arztnetze. Bestandaufnahme und Bewertung im Auftrag des AOKBundesverbandes. Bonn: AOK-Bundesverband; 2002.

46. Stock J. AOK und arztnetze - wohin geht die Reise? Bonn: AOK-Bundesverband Stabsbereich Medizin; 2000.
47. Simoens S, Scott A. The determinants of integrated primary care organizations: an empirical study of local health care co-operatives. Aberdeen: Health Economics Research Unit, University of Aberdeen; 2002.

48. Olesen F, Jensen PB, Grinsted P, Henriksen JS. General practitioners as advisers and coordinators in hospital. Qual Health Care 1998; 7:42-7.

49. Gerlinger T. Rote-grüne Gesundheitspolitik - eine Zwischenbilanz. Berlin: Wissenschaftszemtrum Berlin für Sozialforschung; 2002.

50. AOK Baden-Württemberg/KV Nordbaden/Qualinet. Vertrag Qu@linet. Qualitäts - und Kooperations-modell in der hausärztlichen versorgung Rhein-Neckar. Stuttgart/Karlsruhe/Mannheim: AOK Baden-Württemberg/KV Nordbaden/Qualinet; 2003.

51. Dale J, Lang H, Roberts JA, Green J, Glucksman E. Cost effectiveness of treating care patients in accident and emergency: a comparison between general practitioners, senior house officers and registers. BMJ 1996; 312:1340-4.

52. Busse R. Disease management programs in Germany's Statutory Health Insurance System. Health Aff $2004 ; 23: 56-67$.

53. Altenstter C, Björkman JM. Health policy reform, national variation and globalization. London: MacMillan Press; 1997.

54. Giovanella L. Redes integradas, programas de gestão clínica e clínico geral-coordenador: reformas recentes do setor ambulatorial na Alemanha. In: Anais do I Congresso de Economia da Saúde da América Latina e Caribe [CD-ROM]. Rio de Janeiro: Associação Brasileira de Economia da Saúde; 2004.

55. Saltman R. Drawing the strands together: primary care in perspective. In: Saltman R, Rico A, Boerma W, editors. Primary care in the driver's seat? Berkshire: Open University Press; 2005. p. 68-82.

56. Bodenheimer T, Lo B, Casalino L. Primary care physicians should be coordinators, not gatekeepers. JAMA 1999; 281:2045-9.

57. Saltman R. Melting public-private boundaries in European health systems. Eur J Public Health $2003 ; 13: 24-9$.

58. Rico A. Organizational re-estructuring in European health care: interorganizational coordination and the role of primary care. In: Meeting of the European EU Cost A-15 Network. Welfare Reforms for the 21 st Century. Oslo: European Observatory on Health Care System; 2002.

Recebido em 29/Mar/2005 Aprovado em 02/Ago/2005 